\title{
A Hybrid Power Series Artificial Bee Colony Algorithm to Obtain a Solution for Buckling of Multiwall Carbon Nanotube Cantilevers Near Small Layers of Graphite Sheets
}

\author{
Aminreza Noghrehabadi, ${ }^{1}$ Mohammad Ghalambaz, ${ }^{1}$ \\ Mehdi Ghalambaz, ${ }^{2}$ and Afshin Ghanbarzadeh ${ }^{1}$ \\ ${ }^{1}$ Department of Mechanical Engineering, Shahid Chamran University of Ahvaz, 6135743337 Ahvaz, Iran \\ ${ }^{2}$ Engineering Part of Iman Madar Naslaha Co. (IMEN), Ahvaz, Iran \\ Correspondence should be addressed to Aminreza Noghrehabadi, a.r.noghrehabadi@scu.ac.ir \\ Received 24 April 2012; Revised 13 June 2012; Accepted 17 June 2012 \\ Academic Editor: Erich Klement
}

Copyright (C) 2012 Aminreza Noghrehabadi et al. This is an open access article distributed under the Creative Commons Attribution License, which permits unrestricted use, distribution, and reproduction in any medium, provided the original work is properly cited.

\begin{abstract}
A hybrid power series and artificial bee colony algorithm (PS-ABC) method is applied to solve a system of nonlinear differential equations arising from the distributed parameter model of multiwalled carbon nanotube (MWCNT) cantilevers in the vicinity of thin and thick graphite sheets subject to intermolecular forces. The intermolecular forces are modeled using van der Waals forces. A trial solution of the differential equation is defined as sum of two polynomial parts. The first part satisfies the boundary conditions and does contain two adjustable parameters. The second part is constructed as not to affect the boundary conditions, which involves adjustable parameters. The ABC method is applied to find adjustable parameters of trial solution (in first and second part). The obtained results are compared with numerical results as well as analytical solutions those reported in the literature. The results of the presented method represent a remarkable accuracy in comparison with numerical results. The minimum initial gap and the detachment length of the actuator that does not stick to the substrate due to the intermolecular forces, as important parameters in pull-in instability of MWCNT actuator, are evaluated by obtained power series.
\end{abstract}

\section{Introduction}

Multiwalled carbon nanotubes (MWCNTs) have attracted considerable attention among other nanomaterials because of the potential advantages on markedly improved stiffness, strength, and elimination of main failure mechanism [1]. These novel materials can usually be visualized as nanoscale concentric cylinders rolled up by graphene sheets. MWCNTs are produced by different techniques such as chemical vapor deposition, laser ablation, and arc discharge $[1,2]$. The nanotubes can provide various ranges of conductive properties depending on their atomic and geometrical structure [3]. The unusual properties of MWCNTs have motivated worldwide engineers to explore their applications in different fields of science [4].

Experimental investigations show that the conductance of CNTs is strongly influenced by the occurrence of buckling
[5]. The repeatable transformation between the buckled state and normal state of CNTs produces good potential applications to create devices such as nanotransistors [5], nano-valve, and so forth, [6]. With recent growth in nanotechnology, MWCNTs are increasingly used in developing atomic force microscope (AFM) probes $[1,3,7,8]$ and nanoelectromechanical system (NEMS) switches [9-11].

In the nanoscale, the surface forces play an important role in the design and operation of the MEMS and NEMS devices. The van der Waals force and Casimir force are two significant forces in the scale of nanometers [12]. These forces are basically electromagnetic in nature, and they are important when the separation space between objects is very small [12].

In general, there are two basic approaches to understand the behaviors of CNTs: one is atomistic molecular dynamics simulation and the second is continuum mechanics. 
However, the molecular dynamic method is very time consuming and computationally expensive for a large-scale system.

In a recent work, Koochi et al. [13] applied a hybrid continuum model to investigate the molecular force-induced buckling of a freestanding MWCNT probes/actuators suspended over graphite sheets. They carried out a fourth order nonlinear ordinary differential equation for buckling of multiwalled carbon nanotube (MWCNT) probes/actuators. Koochi et al. [13] used Adomian decomposition method to obtain a solution for buckling and pull-in in stability of MWCNTs. Although, the results of Adomian decomposition method in comparison with numerical results are acceptable, but the results show that the accuracy of Adomian method near the pull-in instability conditions is decreased.

Many different methods have been developed to solve differential equations. However, the solution of nonlinear differential equations still is a challenge $[14,15]$. Recently, artificial intelligence techniques are used to solve nonlinear differential equations and modeling engineering problems [16-21]. Lee and Kang [22] used parallel processor computers to obtain a trial solution for a first order differential equation. Meade and Fernandez [23] applied feed forward neural networks to solve linear and nonlinear ordinary differential equations. Lagaris et al. [15] introduced a new method to solve First order linear ordinary and partial differential equations using artificial neural networks. Malek and Beidokhti [24] applied a hybrid artificial neural networkNelder-Mead optimization technique to solve high order linear differential equations. A hybrid artificial neural networkswarm intelligence method was used by Khan et al. [14] to solve a nonlinear differential equation.

The goal of an optimization problem can be stated as finding the combination of parameters (independent variables) which maximizes or minimizes the value of one or more dependent variables. The value or function to be optimized is called objective function.

Artificial bee colony (ABC) is one of the optimization algorithms, which is introduced by Karaboga in 2005 [25]. The motivation of this algorithm is the intelligent behavior of honey bees. ABC is a simple method with a few main common control parameters such as colony size and maximum cycle number.

Karaboga and Basturk [26] introduced artificial bee colony as an efficient algorithm for numerical function optimization. Karaboga and Akay [27] performed a comparative study on the ABC. They used ABC for optimizing a large set of numerical test functions. They compared the produced results of $\mathrm{ABC}$ algorithm with the results obtained by genetic algorithm, particle swarm optimization algorithm, differential evolution algorithm, and evolution strategies. They reported that the performance of the $A B C$ is better than or similar to those of other population-based algorithms with the advantage of employing fewer control parameters.

In the present study, a combination of power series and artificial bee colony optimization algorithm is applied to obtain a power series solution for the nonlinear ordinary differential equations of MWCNT cantilevers. A remarkable accuracy for the presented method is achieved when the obtained results are compared with numerical results.

\section{Mathematical Model}

Figure 1 shows the schematic of a typical freestanding MWCNT near a surface consisted of $N$ graphene layers, with interlayer distance $d=3.35 \AA$. Consider a MWCNT with the mean radius of $R_{W}$, the length of $L$, and multiwall nanotube layers of $N_{W}$. The gap between MWCNT and the surface is D.

2.1. Electrostatic Domain. Based on continuum mechanics, an MWCNT is modeled by concentric cylindrical tubes. $E_{\text {eff }}$ is Young's modulus of MWCNT and the cross-sectional moment of inertia $I$ is equal to $\pi\left(R_{o}^{4}-R_{i}^{4}\right) / 4$ [13]. By applying the Euler theory and neglecting the effect of large displacement (finite kinematics) for $L / D_{e}>10$ [28], the governing equation of a cantilever MWCNT can be defined in the form of following boundary value differential equation [13]:

$$
E_{\mathrm{eff}} I \frac{d^{4} U}{d X^{4}}=q_{v d W}(D-U)
$$

subject to geometrical and natural boundary conditions as

$$
U(0)=\frac{d U}{d X}(0)=0, \quad \frac{d^{2} U}{d X^{2}}(L)=\frac{d^{3} U}{d X^{3}}(L)=0,
$$

where $X$ is the position along MWCNT measured from the bended end, $U$ is the deflection of MWCNT, and $q_{v d w}$ denote the intermolecular force per unit length of MWCNT. The intermolecular force of $q_{W d v}$ base on double-volume integral of Lennard-Jones potential and some simplification can be represented as

$$
q_{W d v}=\frac{4 C_{6} \sigma^{2} \pi^{2} N N_{W} R_{W}}{d(D-U+N d / 2)^{4}}
$$

for small number of graphene layers [13].

In the above equation, $C_{6}=15.2 \mathrm{eV} \AA^{6}$ is the attractive constants for the carbon-carbon interaction [29], and $\sigma \approx$ $38 \mathrm{~nm}^{-2}$ is the graphene surface density [30]. By substituting (2) in (1a), (1b) and using the following substitutions

$x=\frac{X}{L}, \quad u=\frac{U}{D+N d / 2}, \quad f_{n}=\frac{4 C_{6} \sigma^{2} \pi^{2} N N_{W} R_{W} L^{4}}{E_{\mathrm{eff}} I(D+N d / 2)^{6}}$,

the dimensionless form of (1a), (1b) can be obtained as follows:

$$
\begin{array}{cl}
\frac{d^{4} u}{d x^{4}}=\frac{f}{(1-u(x))^{5}} \\
u(0)=u^{\prime}(0)=0, & \text { at } x=0, \\
u^{\prime \prime}(1)=u^{\prime \prime \prime}(1)=0, & \text { at } x=1,
\end{array}
$$

where in all equations prime denotes differentiation with respect to $x$. 


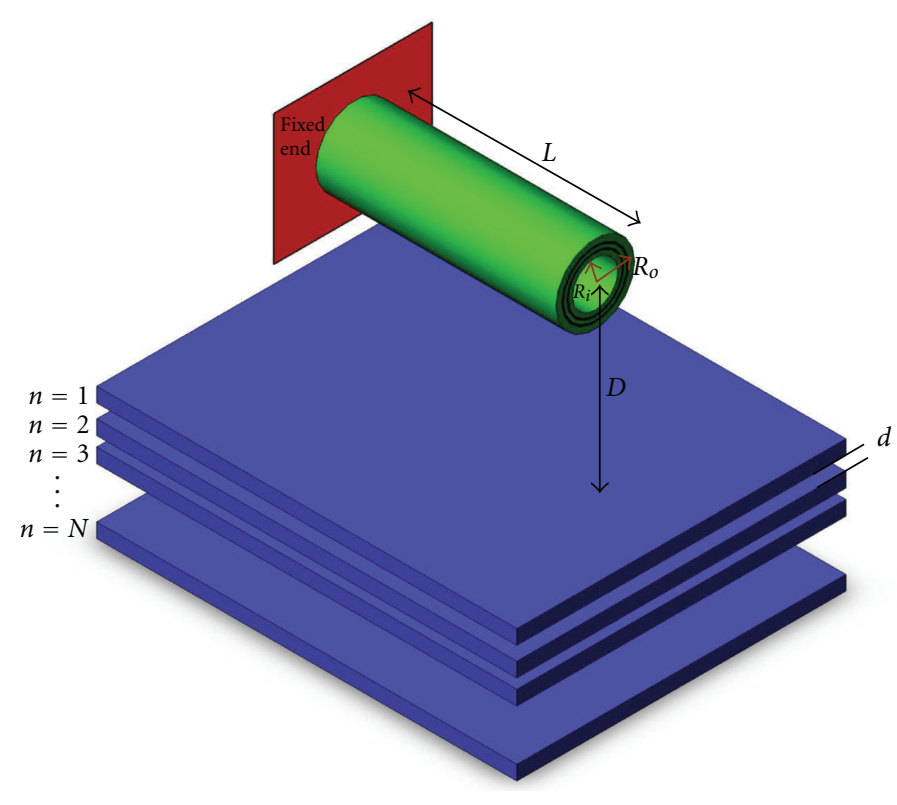

FIgURE 1: Schematic of an MWCNT suspended over graphite sheets.

\section{Artificial Bee Colony Optimization Algorithm}

The artificial bee colony $(\mathrm{ABC})$ algorithm was proposed by Karaboga in 2005. This method is a gradient free optimization algorithm for real parameter optimization, which inspired from intelligent foraging behavior of a bee colony [25].

In a natural bee colony, some tasks are performed by specialized individuals. In the $\mathrm{ABC}$ algorithm, the colony of artificial bees contains three groups of bees as the employed bees, the onlookers, and the scouts [26]. The onlooker bee is a bee waiting for making decision to choose a nectar source. A bee which is going to the nectar source which it previously visited is an employed bee. A scout bee carries out a random search around the hive. In the $\mathrm{ABC}$ algorithm, first half of the colony consists of employed artificial bees and the second half constitutes the onlooker bees. In the method proposed by Karaboga, the number of employed bees is equal to the number of food sources around the hive. The employed bee whose food source is exhausted by the employed and onlooker bees becomes a scout bee. The main steps of the algorithm can be summarized as [26].

(i) Initializing the population which can be strategic or random.

(ii) Repeat procedure:

(a) place the employed bees on the nectar sources in the memory;

(b) place the onlooker bees on the nectar sources in the memory;

(c) send the scouts to the search area for discovering new nectar sources.

(iii) Until requirements are met.
These specialized bees try to maximize the nectar amount stored in the hive by performing efficient division of labor and self-organization. The basic idea and details about $A B C$ algorithm are explained in $[25-27,31]$. In the $A B C$ algorithm, proposed by Karaboga, the position of a nectar source represents a possible solution in the search space for the optimization problem, and the nectar amount of a food source represents the profitability (fitness) of the associated solution.

\section{Solution Method}

The governing equation of a nanotube cantilever was expressed by (1a), (1b). In order to solve (1a), (1b), assume a discretization of the domain $D$ with $m$ arbitrary points. Here, the problem can be written as the following set of equations:

$$
\frac{d^{4} u\left(x_{i}\right)}{d x^{4}}-\left(\frac{f}{\left(1-u\left(x_{i}\right)\right)^{5}}\right)=0, \quad x_{i} \in D, i=1,2, \ldots, m
$$

subject to given boundary conditions in (2).

Let us assume $y_{T}(x, \vec{v})$ as an approximate solution to (1a), (1b) in which $\vec{v}$ is a vector containing adjustable parameters. These parameters (i.e., the adjustable parameters) can be evaluated by minimizing the following sum of squared errors, subject to given boundary conditions in (2)

$$
\begin{array}{r}
E(\vec{a})=\sum_{i=1}^{m}\left(\frac{d^{4} y_{T}\left(x_{i}, \vec{v}\right)}{d x^{4}}-\frac{f}{\left(1-y_{T}\left(x_{i}, \vec{v}\right)\right)^{5}}\right)^{2}, \\
x_{i} \in\left[\begin{array}{ll}
0 & 1
\end{array}\right] .
\end{array}
$$


In order to transform (5) to an unconstrained problem, $y_{T}(x, \vec{a})$ can be written as

$$
\begin{aligned}
Y_{T}(x, \vec{v})= & v_{1} x^{5}+v_{2} x^{4}-\left(10 v_{1}-4 v_{2}\right) x^{3} \\
& +\left(20 v_{1}+6 v_{2}\right) x^{2}+x^{2}(x-1)^{4} N(x, \vec{v})
\end{aligned}
$$

where $v_{1}$ and $v_{2}$ are adjustable parameters. $N(x, \vec{a})$ is a power series $\left(N(x, \vec{v})=\sum_{i=0}^{n} v_{i+3} x^{i}\right)$ which involves adjustable parameters of $v_{3}$ to $v_{n}$. Here, (7) is in the form of a power series with adjustable coefficients, and it exactly satisfies the given boundary conditions of (1-b).

Now, an optimization technique can be applied in order to determine the optimal adjustable parameters of $y_{T}(x, \vec{v})$ (i.e., $\vec{v})$ to minimize $E(\vec{v})$ in (6). Here, the ABC algorithm is used to evaluate the adjustable vector parameter of $v$ to minimize (6).

For different sizes of the series $(n=5,6,7, \ldots, 10)$, the domain of solution is divided to 21 collocation points with equal spices of $0.05\left(x_{i} \in\{0,0.05,0.01 \ldots 1\}\right)$. This configuration was applied for all solutions in the following text. The control parameters of $\mathrm{ABC}$ are essential for obtaining an accurate solution. Increase of colony size increases the calculation time, and decrease of colony size decreases the accuracy of solution and may lead to trap in the local optimums. Table 1 shows the best obtained combination of user-specified parameters of ABC method, which are used for this problem in the following text. This combination of parameters is obtained by trial and error.

In order to verify the convergence and accuracy of the present method, buckling of a typical nanotube-actuator with $f=0.5$ is computed for different size of series using PS-ABC method. The obtained results are compared with the numerical data as well as Adomian decomposition results reported by Koochi et al. [13]. Numerical results are obtained using Maple commercial software, which uses a combination of trapezoid as base scheme and Richardson extrapolation as enhancement scheme $[32,33]$. In the case of $f_{n}=0.5$, the tip deflection was numerically obtained as $u_{t}=0.08323$. The variation of the nanotube cantilever tip deflection $\left(u_{\text {tip }}\right)$, using different selected terms of series is shown in Table 2. This table ensures the convergence and accuracy of the results. As seen, higher accuracy can be obtained by evaluating more terms of the solution $u(x)$. The relative error is computed from

$$
\text { Error }=\left|\frac{u_{\text {Analytical }}-u_{\text {Numerical }}}{u_{\text {Numerical }}}\right| \text {, }
$$

where $u_{\text {Analytical }}$ and $u_{\text {Numerical }}$ are the cantilever MWCNT tip deflection computed from analytical method and the tip deflection computed using numerical method, respectively. Here, error indicates the relative error.

The results of Table 2 show that the PS-ABC with series size of eight almost converged to the numerical result. Thus, the power series size of eight has been selected in the following text for convenience. Comparing this error with the same series size of Adomian method (i.e., eight terms and relative error of $0.12 \%$ ) shows that the PS-ABC method could compute deflection of cantilever MWCNTs with more

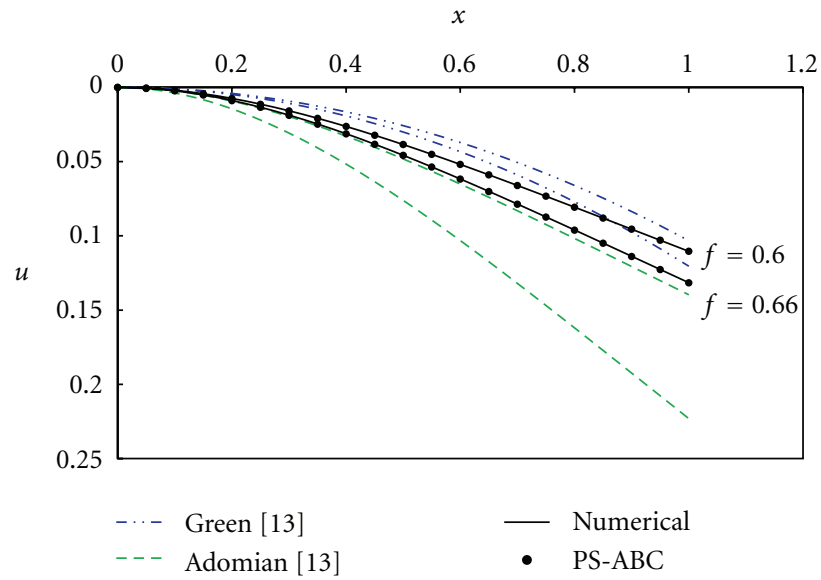

FIGURE 2: Buckling of MWCNT cantilever in the vicinity of graphite sheets for different values of $f$.

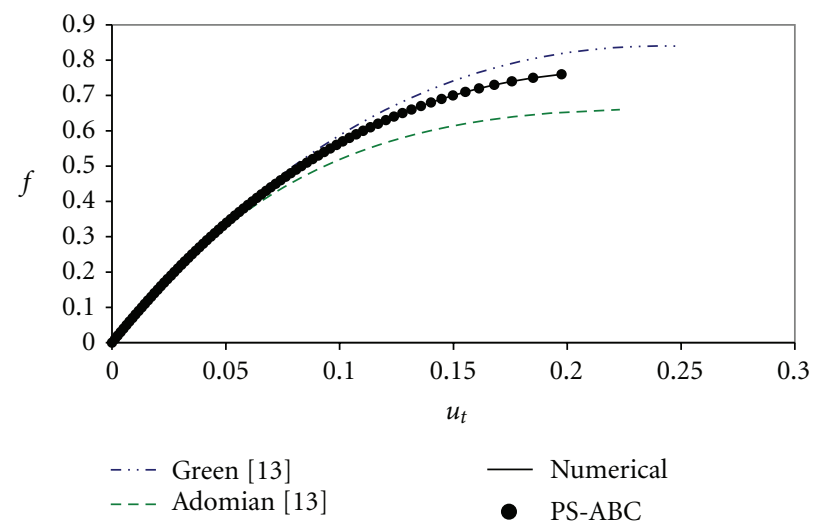

Figure 3: Comparison between the tip buckling of MWCNT cantilevers obtained by different methods.

accuracy than Adomian method. The obtained power series with eight term for $f=0.5$ is as follows:

$$
\begin{aligned}
u(x)= & 0.160624628 x^{2}-0.097464959 x^{3}+0.018038276 x^{4} \\
& +0.000022094 x^{5}+0.003837749 x^{6} \\
& -0.002404155 x^{7}+0.000580025 x^{8} .
\end{aligned}
$$

\section{Results and Discussion}

Figure 2 shows the centerline deflection of nanotubes for selected values of parameter $f$.

This figure reveals that when the parameter of $f$ is small, the buckling can be neglected, and by increase of $f$, the beam deflects into the substrate. As seen, the power series solution in comparison with the same size of Adomian series solution (i.e., [13]) is more powerful to simulate the deflection and instability of nanocantilever beams. Figure 3 shows the variation of tip deflection as a function of $f$. Figures 2 and 3 reveal that the Adomian method underestimated the bucking of nanotubes and Greens method overestimated it while the 
TABLE 1: Control parameters of ABC algorithm.

Control parameter

Value

Type of initial population

Random

The number of colony size (employed bees + onlooker bees)

150 bees

The food source limit which will be abandoned if no improvement was observed

100 try

The maximum foraging try

450 try

TABLE 2: A comparison between the evaluated tip deflection of a typical MWCNT cantilever using different terms of PS-ABC method and Adomian decomposition method for $f=0.5$.

\begin{tabular}{lcccc}
\hline Series size & Tip deflection adomian [13] & Error & Tip deflection PS-ABC & Error \\
\hline 5 & 0.06250 & $2.491 E-01$ & 0.08278 & $5.50 E-03$ \\
6 & 0.09533 & $1.453 E-01$ & 0.08319 & $5.13 E-04$ \\
7 & 0.07516 & $9.700 E-02$ & 0.08324 & $6.41 E-05$ \\
8 & 0.09350 & $1.233 E-01$ & 0.08323 & 0 \\
9 & 0.07737 & $7.046 E-02$ & 0.08323 & 0 \\
10 & 0.08842 & $6.228 E-02$ & 0.08323 & 0 \\
\hline
\end{tabular}

presented method obtained buckling of nanotubes with very good accuracy in comparison with numerical results.

\section{Conclusions}

In this paper, an integration of power series and artificial bee colony optimization method has been utilized in order to obtain a solution for buckling of MWCNT cantilevers subject to small number of graphite layers. The governing differential equation is forth order and highly nonlinear due to the inherent of the van der Waals and electrostatic interactions. A trial solution which exactly satisfies the boundary conditions in the form of a power series with adjustable parameters was introduced. The artificial bee colony optimization algorithm was successfully applied to justify the adjustable parameters of the trial solution. The results of present method are compared with numerical results as well as Adomian decomposition method and Green's method reported in the literature. It is found that the accuracy of present method remarkably is better than the same size of Adomian power series. Therefore, the PS$\mathrm{ABC}$ method can provide an accurate and stable solution for study of MWCNT cantilevers. The present method can be easily extended to solve other nonlinear boundary value differential equations. The future work can be focused on the comparison between the efficiently of the $\mathrm{ABC}$ method and other available optimization methods to tackle the present boundary value differential equation.

\section{Acknowledgments}

The authors are grateful to Shahid Chamran University of Ahvaz for its support through this paper.

\section{References}

[1] A. M. K. Esawi and M. M. Farag, "Carbon nanotube reinforced composites: potential and current challenges," Materials and Design, vol. 28, no. 9, pp. 2394-2401, 2007.
[2] S. J. Chowdhury and B. Howard, "Thermo-mechanical properties of graphite-epoxy composite," International Review of Mechanical Engineering, vol. 4, no. 6, pp. 785-790, 2010.

[3] C. Li, E. T. Thostenson, and T. W. Chou, "Sensors and actuators based on carbon nanotubes and their composites: a review," Composites Science and Technology, vol. 68, no. 6, pp. 1227-1249, 2008.

[4] M. S. Dresselhaus, G. Dresselhaus, and A. Jorio, "Unusual properties and structure of carbon nanotubes," Annual Review of Materials Research, vol. 34, pp. 247-278, 2004.

[5] H. W. C. Postma, T. Teepen, Z. Yao, M. Grifoni, and C. Dekker, "Carbon nanotube single-electron transistors at room temperature," Science, vol. 293, no. 5527, pp. 76-79, 2001.

[6] M. Grujicic, G. Cao, and W. N. Roy, "Computational analysis of the lattice contribution to thermal conductivity of singlewalled carbon nanotubes," Journal of Materials Science, vol. 40, no. 8, pp. 1943-1952, 2005.

[7] S. Akita, "Nanotweezers consisting of carbon nanotubes operating in an atomic force microscope," Applied Physics Letters, vol. 79, pp. 1591-1593, 2001.

[8] Y. Cao, Y. Liang, S. Dong, and Y. Wang, "A multi-wall carbon nanotube (MWCNT) relocation technique for atomic force microscopy (AFM) samples," Ultramicroscopy, vol. 103, no. 2, pp. 103-108, 2005.

[9] M. Paradise and T. Goswami, "Carbon nanotubesproduction and industrial applications," Materials and Design, vol. 28, no. 5, pp. 1477-1489, 2007.

[10] R. H. Baughman, C. Cui, A. A. Zakhidov et al., "Carbon nanotube actuators," Science, vol. 284, no. 5418, pp. 13401344, 1999.

[11] C. H. Ke, N. Pugno, B. Peng, and H. D. Espinosa, "Experiments and modeling of carbon nanotube-based NEMS devices," Journal of the Mechanics and Physics of Solids, vol. 53, no. 6, pp. 1314-1333, 2005.

[12] G. L. Klimchitskaya, E. V. Blagov, and V. M. Mostepanenko, "Van der Waals and Casimir interactions between atoms and carbon nanotubes," Journal of Physics A, vol. 41, no. 16, Article ID 164012, 2008.

[13] A. Koochi, A. S. Kazemi, A. Noghrehabadi, A. Yekrangi, and M. Abadyan, "New approach to model the buckling and stable 
length of multi walled carbon nanotube probes near graphite sheets," Materials and Design, vol. 32, no. 5, pp. 2949-2955, 2011.

[14] J. A. Khan, R. M. A. Zahoor, and I. M. Qureshi, "Swarm Intelligence for the problems of non-linear ordinary differential equations and its application to well known Wessinger's equation," European Journal of Scientific Research, vol. 34, no. 4, pp. 514-525, 2009.

[15] I. E. Lagaris, A. Likas, and D. I. Fotiadis, "Artificial neural networks for solving ordinary and partial differential equations," IEEE Transactions on Neural Networks, vol. 9, no. 5, pp. 9871000, 1998.

[16] M. Ghalambaz, A. R. Noghrehabadi, M. A. Behrang, E. Assareh, A. Ghanbarzadeh, and N. Hedayat, "A hybrid neural network and gravitational search algorithm (HNNGSA) method to solve well known Wessinger's equation," Proceedings of World Academy of Science, Engineering and Technology, vol. 73, pp. 803-807, 2011.

[17] A. Noghrehabadi, M. Ghalambaz, and M. Ghalambaz, "A hybrid power series - artificial bee colony to solve magnetohydrodynamic viscous flow over a nonlinear permeable shrinking sheet," International Review on Modelling and Simulations, vol. 4, no. 5, pp. 2696-2700, 2011.

[18] M. A. Behrang, M. Ghalambaz, E. Assareh, and A. R. Noghrehabadi, "A new solution for natural convection of darcian fluid about a vertical full cone embedded in porous media prescribed wall temperature by using a hybrid neural networkparticle swarm optimization method," World Academy of Science, Engineering and Technology, vol. 73, pp. 1108-1113, 2011.

[19] E. Assareh, M. A. Behrang, M. Ghalambaz, A. R. Noghrehabadi, and A. Ghanbarzadeh, "A New Approach to Solve Blasius Equation using Parameter Identification of Nonlinear Functions based on the Bees Algorithm (BA), vol. 73, World Academy of Science, Engineering and Technology, 2001.

[20] M. Ghalambaz, A. Noghrehabadi, and A. Vosoogh, "A hybrid Power series-Artificial Bee Colony algorithm to solve electrostatic pull-in instability and deflection of nano cantilever actuators considering Casimir Attractions," International Review of Mechanical Engineering, vol. 5, no. 4, 2011.

[21] A. Yekrangi, M. Ghalambaz, A. Noghrehabadi et al., "Approximate solution for a simple pendulum beyond the small angles regimes using hybrid artificial neural network and particle swarm optimization algorithm," Procedia Engineering, vol. 10, pp. 3742-3748, 2011.

[22] H. Lee and I. S. Kang, "Neural algorithm for solving differential equations," Journal of Computational Physics, vol. 91, no. 1, pp. 110-131, 1990.

[23] A. J. Meade Jr. and A. A. Fernandez, "The numerical solution of linear ordinary differential equations by feedforward neural networks," Mathematical and Computer Modelling, vol. 19, no. 12, pp. 1-25, 1994.

[24] A. Malek and R. S. Beidokhti, "Numerical solution for high order differential equations using a hybrid neural networkoptimization method," Applied Mathematics and Computation, vol. 183, no. 1, pp. 260-271, 2006.

[25] D. Karaboga, "An idea based on honey bee swarm for numerical optimization," Tech. Rep. TR06, Erciyes University, Engineering Faculty, Computer Engineering Department, 2005.

[26] D. Karaboga and B. Basturk, "A powerful and efficient algorithm for numerical function optimization: Artificial Bee Colony (ABC) algorithm," Journal of Global Optimization, vol. 39, no. 3, pp. 459-471, 2007.
[27] D. Karaboga and B. Akay, "A comparative study of Artificial Bee Colony algorithm," Applied Mathematics and Computation, vol. 214, no. 1, pp. 108-132, 2009.

[28] W. H. Lin and Y. P. Zhao, "Casimir effect on the pull-in parameters of nanometer switches," Microsystem Technologies, vol. 11, no. 2-3, pp. 80-85, 2005.

[29] L. A. Girifalco, M. Hodak, and R. S. Lee, "Carbon nanotubes, buckyballs, ropes, and a universal graphitic potential," Physical Review B, vol. 62, no. 19, pp. 13104-13110, 2000.

[30] M. Dequesnes, S. V. Rotkin, and N. R. Aluru, "Calculation of pull-in voltages for carbon-nanotube-based nanoelectromechanical switches," Nanotechnology, vol. 13, no. 1, pp. 120-131, 2002.

[31] B. Akay and D. Karaboga, "A modified Artificial Bee Colony algorithm for real-parameter optimization," Information Sciences, vol. 192, pp. 120-142, 2012.

[32] U. Ascher, R. Mattheij, and R. Russell, Numerical Solution of Boundary Value Problems for Ordinary Differential Equations, vol. 13 of SIAM Classics in Applied Mathematics, 1995.

[33] U. Ascher and L. Petzold, Computer Methods for Ordinary Differential Equations and Differential-Algebraic Equations, SIAM, Philadelphia, Pa, USA, 1998. 

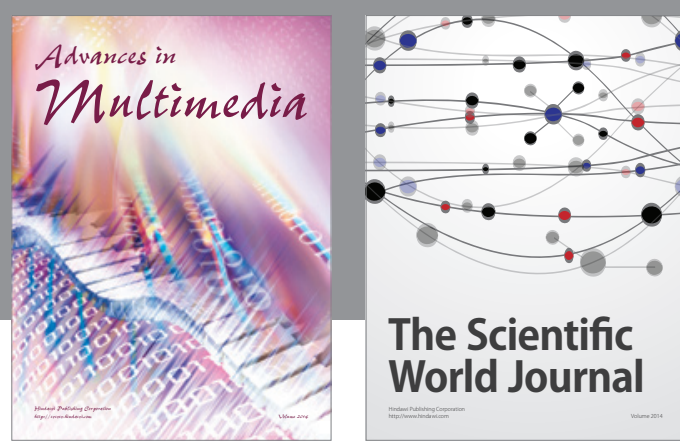

The Scientific World Journal
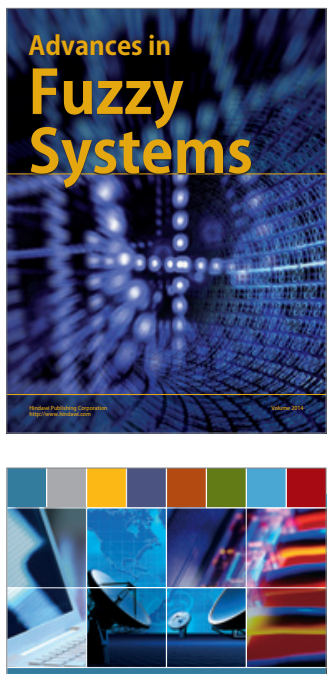

Computer Networks and Communications
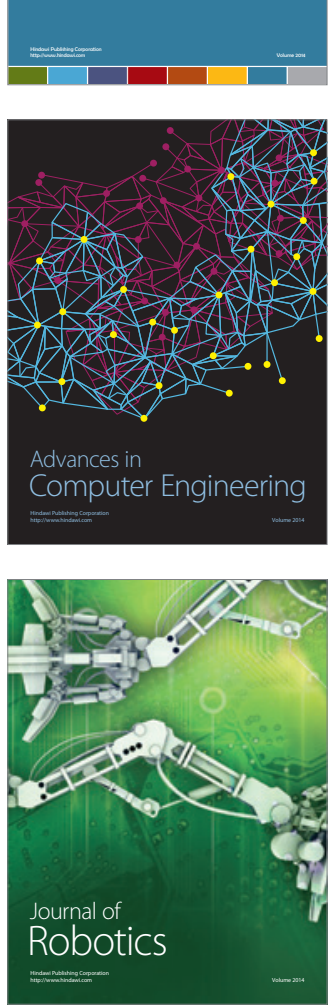
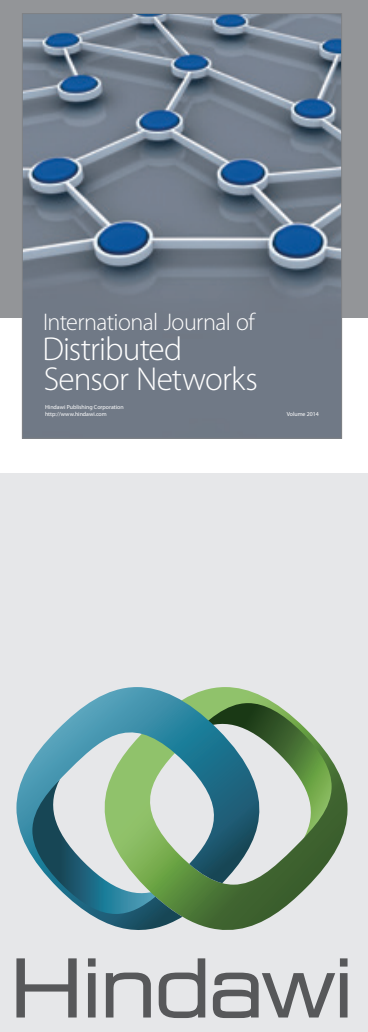

Submit your manuscripts at

http://www.hindawi.com
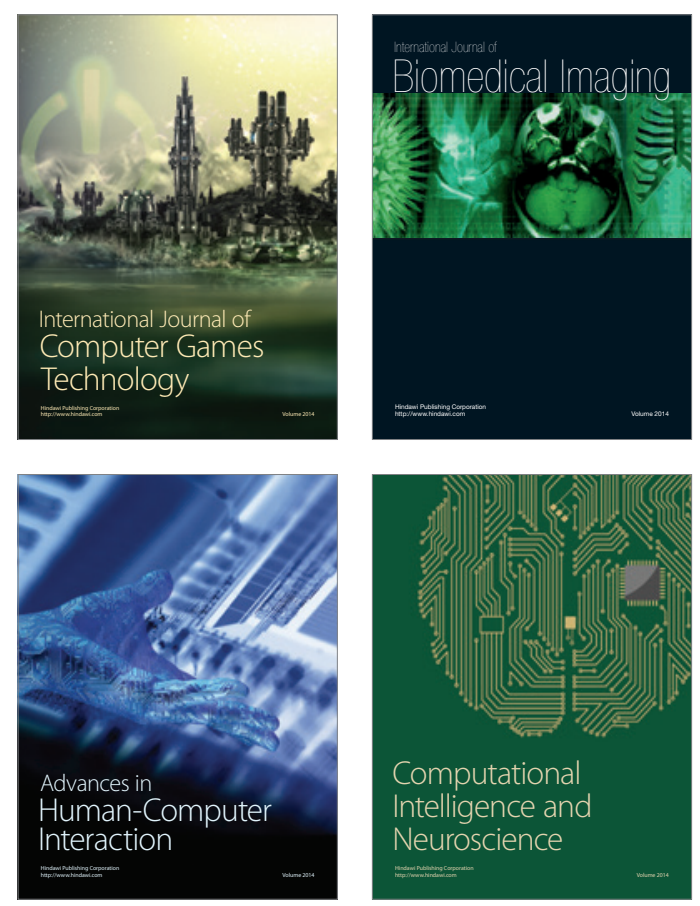
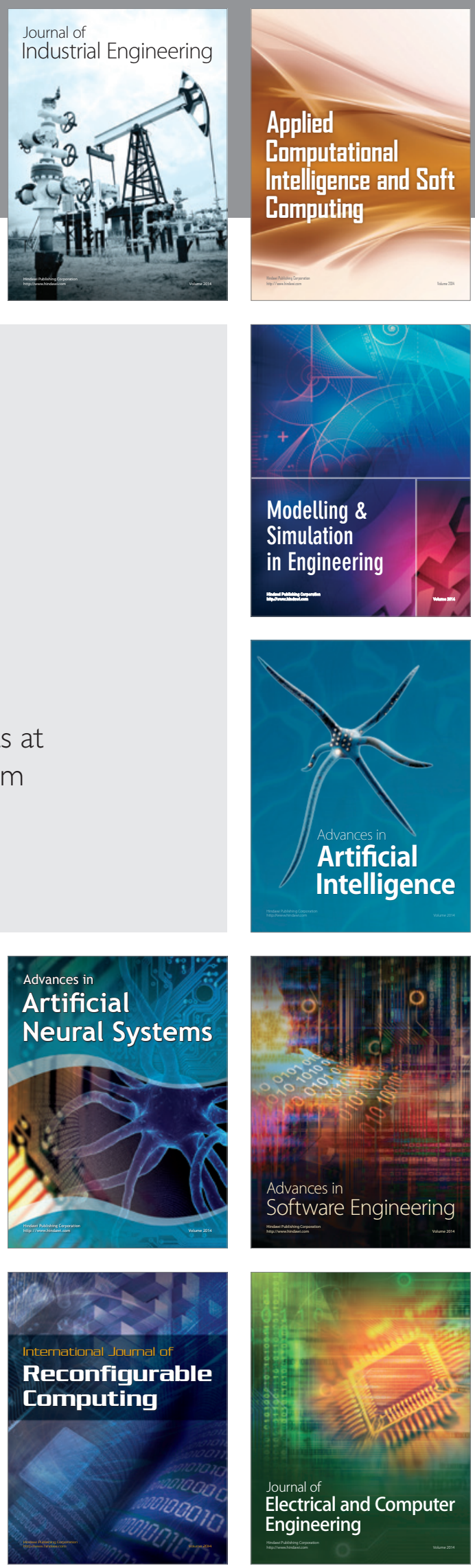